\title{
A comparison of inverse transform and composition methods of data simulation from the Lindley distribution
}

\author{
Macaulay Okwuokenye ${ }^{1, a}$, Karl E. Peace ${ }^{a}$ \\ ${ }^{a}$ Department of Biostatistics, Jiann-Ping Hsu College of Public Health, \\ Georgia Southern University, USA
}

\begin{abstract}
This study compares the inverse transform and the composition methods for generating data from the Lindley distribution. The expression for the inverse of the distribution function for the Lindley distribution does not exist in closed form. Hence, authors of many empirical studies on the Lindley distribution used methods for generating Lindley variates other than the inverse transform. We generated data from the Lindley distribution using the inverse transform approach by obtaining the Lindley variates numerically; we also generated data from this distribution using the composition approach. Following the generation of the Lindley variates using these two methods, we compare some statistical properties of the estimates of the Lindley model parameters based on the generated data. We conclude that the two methods produce similar results.
\end{abstract}

Keywords: Lindley distribution, simulation, inverse transform, composition, mixture density, weighted density, random variable, Lambert function

\section{Introduction}

When conducting empirical study of a random variable, one generates the random variable, $X$, whose distribution function:

$$
F(x)=\operatorname{Pr}(X \leq x), \quad-\infty<x<\infty
$$

is known. Random variables are generated by manipulation of one or more uniform random $U \sim(0,1)$ variables using the desired distribution function in Equation (1.1). The choice of data generation technique is driven by (a) whether the distribution of the desired variates has a closed form, (b) computing time, (c) simplicity of implementation of the chosen algorithm, and (d) needed computer memory of the random number generator (Banks, 1998).

Many methods exist for generating random variables. Common methods include inverse transform, composition, acceptance-rejection, and convolution. Data may also be generated using the Lambert function $W(z)$, which is an inverse function (Chapeau-Blondeau and Monir, 2002) (see Appendix A for application of this function to generating the Lindley variates). Inverse transform and composition methods are of interest in this study; hence, details of other methods are not discussed further.

The rest of the paper is organized as follows: Sections 1.1, 1.2, and 1.3 present brief descriptions of the inverse transform, the composition method, and the Lindley distribution, respectively. In Section 2, we describe generation of Lindley variates using these two methods. Section 3 presents the results and comments; Section 4, conclusions.

\footnotetext{
${ }^{1}$ Corresponding author: Department of Biostatistics, Adjunct Faculty, Jiann-Ping Hsu College of Public Health, Georgia Southern University, Statesboro, GA, USA. E-mail: chiefendorce@ hotmail.com

Published 30 November 2016 / journal homepage: http://csam.or.kr

(C) 2016 The Korean Statistical Society, and Korean International Statistical Society. All rights reserved.
} 


\subsection{Inverse transform method}

Suppose a random variable $X$ with a density function $f(x)$ has a continuous and strictly increasing distribution function $F(x)$. The distribution and density functions are related by

$$
F(x)=\int_{-\infty}^{x} f(w) d w
$$

Denote by $F^{-1}(x)$ the inverse of $F(x)$. The random variable $X$ can be generated as follows:

1. Generate $U$ from $U(0,1)$;

2. return $X=F_{X}^{-1}(U)$. That is, an inverse transformation is used to convert the $U(0,1)$ to a random variate of the desired distribution.

The inverse transform method works because

$$
\operatorname{Pr}(X \leq x)=\operatorname{Pr}\left[F^{-1}(U) \leq x\right]=\operatorname{Pr}[U \leq F(x)] .
$$

The right hand side of Equation (1.2) is the distribution function of a uniform random variable evaluated at $F(x)$.

\subsection{Composition method}

Assume $f(x)$ can be expressed as a weighted sum of $k$ different densities:

$$
f(x)=\sum_{i=1}^{k} p_{i} f_{i}(x), \quad p_{i}>0,
$$

where $\sum_{i=1}^{k} p_{i}=1$ and $f_{i}(x)$ are the component density functions. The constraint $\sum_{i=1}^{k} p_{i}=1$ ensures that the component densities are mutually exclusive and exhaustive. The composite density $f(x)$ can be generated as a mixture of the component densities $f_{i}(x)$ if methods exist for generating random variates from the component densities. The composite density function in Equation (1.3) is often called a mixture or compound density.

\subsection{The Lindley model}

The Lindley distribution (Lindley, 1958) was introduced in connection with fudicial distribution and the Bayes theorem, but it has not seen much utility in the analyses of failure time data until the past decade. Its mathematical properties and real data applications were recently explored by Ghitany et al. (2008). Since then, studies involving the Lindley distribution have become an area of growing interest. The Lindley probability density function (PDF) is

$$
f(t ; \theta)=\frac{\theta^{2}}{1+\theta}(1+t) \exp (-\theta t), \quad t, \theta>0,
$$

where $\theta$ is the scale parameter and $t$, the survival times. The corresponding cumulative distribution and survival functions are

$$
F(t ; \theta)=1-\frac{1+\theta+\theta t}{1+\theta} \exp (-\theta t), \quad t, \theta>0
$$


and

$$
S(t ; \theta)=1-F(t ; \theta)
$$

respectively. Suppose $T_{1}, T_{2}, \ldots, T_{n}$ is a random sample from the Lindley distribution, the estimator for both maximum likelihood estimate (MLE) and method of moments estimate of the parameter $\theta$ is

$$
\hat{\theta}=\frac{-(\bar{T}-1)+\sqrt{(\bar{T}-1)^{2}+8 \bar{T}}}{2 \bar{T}}, \quad \bar{T}>0 .
$$

The estimator $\hat{\theta}$ of $\theta$ is positively biased: $E\{\hat{\theta}\}-\theta>0$; the mathematical proof is presented elsewhere (Ghitany et al., 2008).

Different approaches have been employed to generate data that follow the Lindley distribution when performing empirical studies for assessing operating characteristics of this distribution. These approaches vary in their merits and demerits. The composition approach utilized by Ghitany et al. (2008) is convenient because it generates the Lindley variates as a mixture of two known density functions-the exponential and a special case of the gamma. This may be partly because the Lindley distribution is not yet supported in most available softwares, and partly because the inverse transformation for the Lindley distribution cannot be explicitly expressed. However, this method might pose a challenge when one is interested in incorporating covariate information using the Lindley distribution as the time-dependent component of the Cox proportional hazard model (Cox, 1972; Okwuokenye, 2012; Okwuokenye and Peace, 2015b). This is because whereas the exponential distribution is a proportional hazard model, the gamma distribution is not.

Ghitany et al. (2008) asserts that inversion method fails as a method for generating data from the Lindley distribution because $F(t)=U, U \sim(0,1)$, cannot be explicitly expressed. However, we note that numerical techniques may be employed to solve for the random variable $T$ given $F(T)=U$ when using this method to generate data from a distribution whose inverse transformation cannot be explicitly expressed (Wicklin, 2013). Examples of such numerical techniques are the bisection and Newton-Raphson methods. We are unaware of any empirical comparison of the inverse transform and composition methods for simulating data from the Lindley distribution.

Therefore, in this study, we compare the inverse transform and the composition methods for generating data from the Lindley distribution. Following the generation of the Lindley variates using these two methods, we compared some statistical properties of estimates of Lindley model parameters based on the generated data.

\section{Method}

\subsection{Generating data from the Lindley distribution using inverse transform method}

Suppose $U_{i}(i=1,2, \ldots, n)$ are random numbers, times-to-event $\left(t_{i}\right)$ may be obtained by solving Equation (2.1) of the cumulative hazard $H\left(t_{i}\right)$ for $t_{i}$ :

$$
H\left(t_{i}\right)=\int_{0}^{t_{i}} h_{0}(w) d w=-\ln \left(1-u_{i}\right)
$$

where $h_{0}$ is the hazard rate function, and $U_{i}$ is a uniform random variable. The $t_{i}^{\prime} s$ may be obtained by solving for $t_{i}$ in the non-linear equation that results from replacing $h_{0}$ in Equation (2.1) with the 
Lindley hazard rate function:

$$
h(t ; \theta)=\frac{\theta^{2}(1+t)}{\theta+1+\theta t}, \quad \theta>0, t \geq 0 .
$$

Equation (2.1) has no explicit form for the Lindley distribution. Therefore, a numerical technique, such as the Newton-Raphson, may be employed to solve for the Lindley variates (Bender et al., 2005; Okwuokenye, 2012; Okwuokenye and Peace, 2015a, 2015b; Peace, 1976; Peace and Flora, 1978; Qian et al., 2010; Wicklin, 2013). We generated data from the Lindley distribution using inverse transform by iteratively solving $T=F_{T}^{-1} U$ in Equation (2.2):

$$
\ln \left[\frac{1+\theta+\theta t}{1+\theta} \exp (-\theta t)\right]=\ln (1-u) .
$$

\subsection{Generating data from the Lindley distribution using the composition method}

The expression for inverse of $F(t)$ for the Lindley distribution does not exit in closed form. Ghitany $e t$ al. (2008) notes that Lindley density function can be expressed as a mixture of the exponential $(\theta)$ and gamma $(2, \theta)$ distributions with pdfs $\theta \exp (-\theta t)$ and $\theta^{2} t \exp (-\theta t)$, respectively. The respective mixing proportions are $\theta /(1+\theta)$ and $1 /(1+\theta)$. In particular, the Lindley density function can be expressed as in Equation (2.3):

$$
f(t)=p f_{1}(t)+(1-p) f_{2}(t), \quad t>0, p>0,
$$

where $p=\theta /(1+\theta), f_{1}(t)=\theta \exp (-\theta t)$ and $f_{2}(t)=\theta^{2} t \exp (-\theta t)$.

To generate $t_{i}, i=1,2, \ldots, n$ from the Lindley distribution, the following algorithm (Ghitany $e t$ al., 2008) based on the composition method was used:

1. Generate $U_{i} \sim$ Uniform $(0,1) ; \quad i=1,2, \ldots, n$.

2. Generate $V_{i} \sim \operatorname{Exponential}(\theta) ; \quad i=1,2, \ldots, n$.

3. Generate $W_{i} \sim \operatorname{Gamma}(2, \theta) ; \quad i=1,2, \ldots, n$.

4. If $U_{i} \leq p=\theta /(1+\theta)$ then set $t_{i}=V_{i}$; otherwise $t_{i}=W_{i}, \quad i=1,2, \ldots, n$.

For the two methods under study, $m=10,000$ replicates of data sets following the Lindley distribution are generated by specifying the Lindley parameter $(\theta=0.10,1.00,9.00)$ for different sample sizes $(n=20,40,80,100,200)$. The choice of parameter values were chosen to allow for comparison of results with those of Ghitany et al. (2008). The same seed and pseudo random number generator were used for the two methods. Once the data were generated using the two methods, the same Lindley log-likelihood function was applied to the two generated data sets to obtain MLEs of the Lindley distribution survival parameter. For numerical solution using the inverse transform, convergence was attained when the relative change in gradient between two consecutive iteration was $1 \times 10^{-8}$.

The following measures were computed:

(i) Average bias of estimates of $\hat{\theta}_{i}, i=1,2, \ldots, m$ :

$$
\frac{1}{m} \sum_{i=1}^{m}\left(\hat{\theta}_{i}-\theta\right)
$$


where $\hat{\theta}$ is the estimate of the true parameter $\theta$ of the Lindley distribution. This represents the deviation of the estimates from the parameter values used for simulation.

(ii) Average mean square error (MSE) of the estimates of $\hat{\theta}_{i}, i=1,2, \ldots, m$ :

$$
\frac{1}{m} \sum_{i=1}^{m}\left(\hat{\theta}_{i}-\theta\right)^{2}
$$

(iii) Coverage probability computed as the proportion of $m$ estimated confidence intervals which include the parameter $\theta$.

(iv) Average width of the confidence interval computed as

$$
\frac{1}{m} \sum_{i=1}^{m}\left(\hat{U}_{i}-\hat{L}_{i}\right)
$$

were $\hat{U}$ and $\hat{L}$ represent the upper and lower limits of the estimated Wald-based 95\% confidence interval (CI) of $\hat{\theta}$, respectively. Also computed were the mean of parameter estimates and a $95 \%$ CI obtained by computing the 2.5 and 97.5 percentiles of the distribution of the estimates.

(v) Estimate of type I error

$$
\alpha=\frac{1}{m} \sum z_{i} \times 100,
$$

where $\alpha$ is the proportion of times the Kolmogorov-Smirnov test rejects the null hypothesis of equality of empirical distribution functions. $z_{i}$ denotes the instances when the null hypothesis was rejected; $m$ is the number of replicates. The proportion of times out of $m$ replicates that the Kolmogorov-Smirnov test for two sample rejects the null hypothesis of no difference in empirical distribution functions based on the two methods may be used to estimate the type I error. The Kolmogorov-Smirnov (K-S) statistic measures the maximum deviation of the empirical distribution function (EDF). For an indicator function $I$ (equal to 1 if $X_{i} \leq x$ and equal to 0 otherwise), define the EDF of a sample $\left\{X_{j}\right\}, j=1,2, \ldots n$ as

$$
F(x)=\frac{1}{n}\left(\text { number of } x_{j} \leq x\right)=\frac{1}{n} \sum_{j=1}^{n} I\left(x_{j} \leq x\right) .
$$

The K-S statistics for comparing two samples is expressed as

$$
D=\max _{j}\left|F_{1}\left(x_{j}\right)-F_{2}\left(x_{j}\right)\right|, \quad j=1,2, \ldots, n .
$$

$D$ is the largest distance observed in the empirical quantile-quantile plot when the distribution of a variable is plotted in two different samples. The probability that $D$ is greater than the observed value $d$ under the null hypothesis of no difference between the two EDF is the $p$-value for this test. Because we were comparing two samples with expectedly the same EDF and the same parameter values, we know the null hypothesis that the two EDF are equal was true. Since the considered sample size was relatively small, we used Monte Carlo estimates of exact $p$-values (Agresti et al., 1979). 
Table 1: Average bias (actual deviation and deviation converted to percent of true value) of parameter estimates based on simulated data using composition and inverse transform methods

\begin{tabular}{|c|c|c|c|c|c|c|}
\hline \multirow{2}{*}{$\theta$} & \multirow{2}{*}{ Method } & \multicolumn{5}{|c|}{ Sample size $(n)$} \\
\hline & & 20 & 40 & 80 & 100 & 200 \\
\hline \multirow{4}{*}{0.10} & MM & 0.0026 & 0.0013 & 0.0007 & 0.0005 & 0.0003 \\
\hline & $\overline{d b} / \theta \times 100$ & 2.60 & 1.30 & 0.70 & 0.50 & 0.30 \\
\hline & $\mathrm{HM}$ & 0.0024 & 0.0013 & 0.0006 & 0.0005 & 0.0002 \\
\hline & $\overline{d b} / \theta \times 100$ & 2.40 & 1.30 & 0.60 & 0.50 & 0.20 \\
\hline \multirow{4}{*}{1.00} & MM & 0.0316 & 0.0146 & 0.0094 & 0.0056 & 0.0024 \\
\hline & $\overline{d b} / \theta \times 100$ & 3.16 & 1.46 & 0.94 & 0.56 & 0.24 \\
\hline & $\mathrm{HM}$ & 0.0299 & 0.0158 & 0.0078 & 0.0060 & 0.0028 \\
\hline & $\overline{d b} / \theta \times 100$ & 2.99 & 1.58 & 0.78 & 0.60 & 0.28 \\
\hline \multirow{4}{*}{9.00} & MM & 0.4200 & 0.2034 & 0.1123 & 0.0832 & 0.0341 \\
\hline & $\overline{d b} / \theta \times 100$ & 4.67 & 2.26 & 1.25 & 0.90 & 0.38 \\
\hline & $\mathrm{HM}$ & 0.4040 & 0.2091 & 0.1027 & 0.0794 & 0.0376 \\
\hline & $\overline{d b} / \theta \times 100$ & 4.49 & 2.32 & 1.14 & 0.88 & 0.42 \\
\hline
\end{tabular}

Values of actual deviation, $\bar{d} b=(1 / m) \sum_{i=1}^{m}\left(\hat{\theta}_{i}-\theta\right)$ and deviation converted to percent of true value. HM and MM are simulation method using inverse transform method and mixture of exponential and gamma distributions, respectively. $m=10,000$ replicates.

Table 2: Average mean square error of parameter estimates based on simulated data using composition and inverse transform methods

\begin{tabular}{ccccccc}
\hline \hline \multirow{2}{*}{$\theta$} & \multirow{2}{*}{ Method } & \multicolumn{5}{c}{ Sample size $(n)$} \\
\cline { 3 - 7 } & & 20 & 40 & 80 & 100 & 200 \\
\hline \multirow{2}{*}{0.10} & MM & 0.0003 & 0.00010 & 0.0001 & 0.0001 & 0.00003 \\
& HM & 0.0003 & 0.00018 & 0.0001 & 0.0001 & 0.00003 \\
\hline \multirow{2}{*}{1.00} & MM & 0.0342 & 0.01590 & 0.0076 & 0.0059 & 0.00280 \\
& HM & 0.0337 & 0.01560 & 0.0075 & 0.0061 & 0.00290 \\
\hline \multirow{2}{*}{9.00} & MM & 4.2340 & 1.92780 & 0.9152 & 0.7051 & 0.34820 \\
& HM & 4.3020 & 1.92050 & 0.9121 & 0.7318 & 0.35290 \\
\hline \hline
\end{tabular}

Values of $(1 / m) \sum_{i=1}^{m}\left(\hat{\theta}_{i}-\theta\right)^{2}$. HM and MM are simulation method using inverse transform method and mixture of exponential and gamma distributions, respectively. $m=10,000$ replicates.

\section{Results and comments}

Tables 1 and 2 present results of estimated average bias (actual deviation and deviation converted to percent of true value) and average MSE of $\hat{\theta}$, respectively. The results are consistent with that presented by Ghitany et al. (2008); in fact, majority of the estimates are very similar if rounded off to the same number of decimals. Notably, as expected $\hat{\theta}$ is positively biased and the mean bias decreases as $n$ increases as does the average MSE-which one expects from the CAN property of MLE's.

Table 3 presents the coverage probability $(1-\alpha) \times 100=95 \%$ of the parameter estimates. The coverage probabilities are all close to the target $(95 \%)$ value. In all occasions, the estimates from the mixture and inverse transform method are essentially the same.

Table 4 presents average width of estimated Wald-based $95 \%$ CIs of parameter estimates for simulated data using the composition and the inverse transform methods. Again, the results for the two methods are very similar. Figures 1 and 2 display the Wald-based 95\% CIs of the parameter estimates for the first 150 replicates for some parameter values (and $n=20$ ); the Wald-based 95\% CIs are similar for the two methods. Tabulating the $95 \%$ CIs of the parameter estimates for all the replicates is impractical because of space limitation. Additionally, for composition method with $n=20$, $\theta=0.1$, the center of the CI (i.e., parameter estimate) is above the true parameter value $52.32 \%$ of the time; for the inverse transform method, the parameter estimate is above the true parameter value 
Table 3: Coverage probability of parameter estimates based on simulated data using composition and inverse transform methods

\begin{tabular}{|c|c|c|c|c|c|c|}
\hline \multicolumn{7}{|c|}{ Coverage probability of $\hat{\theta}$} \\
\hline \multirow{2}{*}{$\theta$} & \multirow{2}{*}{ Method } & \multicolumn{5}{|c|}{ Sample size $(n)$} \\
\hline & & 20 & 40 & 80 & 100 & 200 \\
\hline \multirow{2}{*}{0.10} & MM & 96.28 & 96.66 & 95.22 & 95.41 & 95.13 \\
\hline & $\mathrm{HM}$ & 96.24 & 95.77 & 95.09 & 95.18 & 94.94 \\
\hline \multirow{2}{*}{1.00} & MM & 96.52 & 95.62 & 95.46 & 95.52 & 95.38 \\
\hline & HM & 96.25 & 95.80 & 95.09 & 95.17 & 94.98 \\
\hline \multirow{2}{*}{9.00} & MM & 96.32 & 95.93 & 95.50 & 95.13 & 95.25 \\
\hline & HM & 96.29 & 95.80 & 95.13 & 96.94 & 95.00 \\
\hline
\end{tabular}

Values of coverage probability computed as the proportion of the $m$ estimated confidence intervals which include the parameter $\theta$. HM and MM are simulation method using inverse transform method and mixture of exponential and gamma distributions, respectively. $m=10,000$ replicates. Targeted coverage probability is $1-\alpha=0.95 \times 100$.

Table 4: Average width of estimated Wald-based 95\% confidence interval of parameter estimate for simulated data using composition and inverse transform methods

\begin{tabular}{ccccccc}
\hline \hline \multirow{2}{*}{$\theta$} & \multirow{2}{*}{ Method } & \multicolumn{5}{c}{ Sample size $(n)$} \\
\cline { 3 - 7 } & & 20 & 40 & 80 & 100 & 200 \\
\hline \multirow{2}{*}{0.10} & MM & 0.0678 & 0.0458 & 0.03177 & 0.0283 & 0.0198 \\
& HM & 0.0677 & 0.0459 & 0.03174 & 0.0283 & 0.0198 \\
\hline \multirow{2}{*}{1.00} & MM & 0.7305 & 0.4911 & 0.33990 & 0.3018 & 0.2114 \\
& HM & 0.7292 & 0.4917 & 0.33930 & 0.3020 & 0.2115 \\
\hline \multirow{2}{*}{9.00} & MM & 8.0860 & 5.4022 & 3.72090 & 3.3065 & 2.3102 \\
& HM & 8.0715 & 5.4058 & 3.71608 & 3.3050 & 2.3111 \\
\hline \hline
\end{tabular}

Values of $(1 / m) \sum_{i=1}^{m}\left(\hat{U}_{i}-\hat{L}_{i}\right)$ representing width of $95 \%$ confidence interval computed as the difference in the upper and lower estimated Wald-based confidence intervals of $\theta$. HMandMMare simulation method using inverse transform method and mixture of exponential and gamma distributions, respectively. $m=10,000$ replicates. $\hat{U}_{i}$ and $\hat{L}_{i}$ represents the estimated upper and lower limit, respectively, of a $95 \%$ confidence interval.

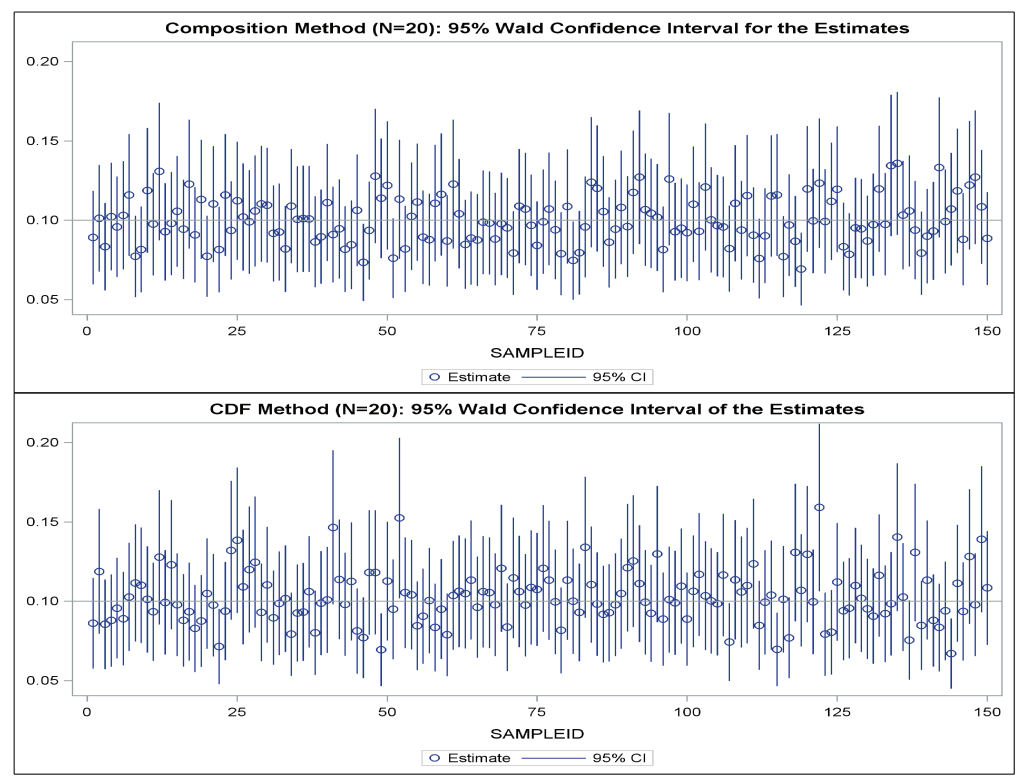

Figure 1: Wald-based 95\% confidence intervals for $n=20 ; \theta=0.1$. 


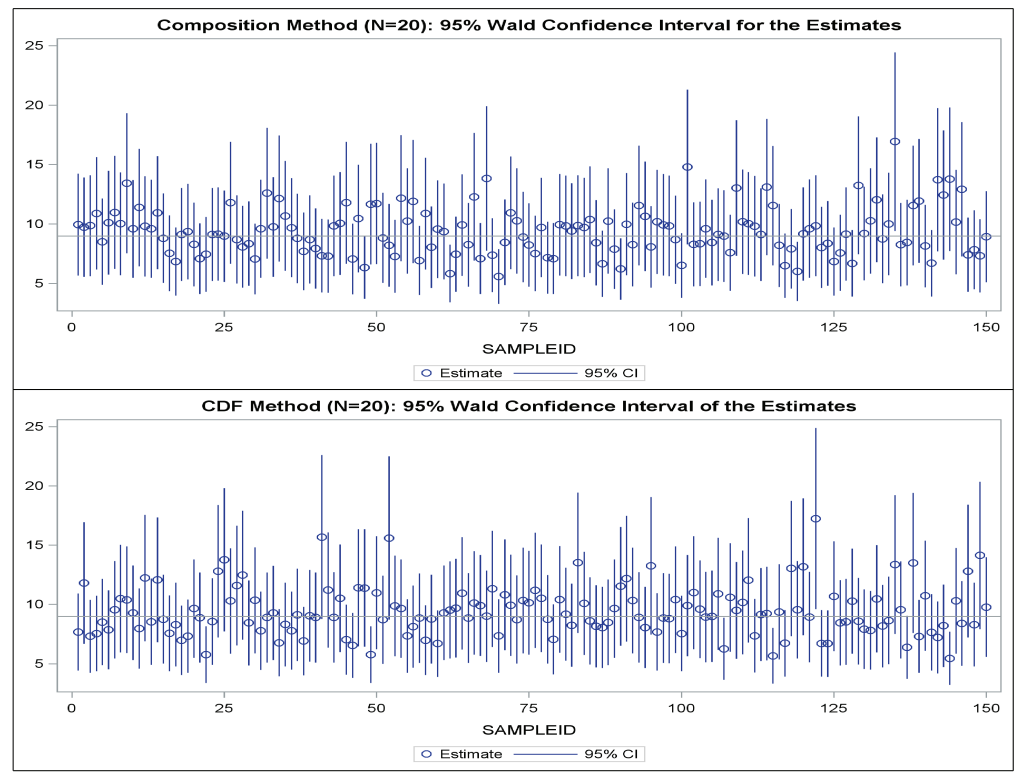

Figure 2: Wald-based 95\% confidence interval for $n=20 ; \theta=9$.

$52.11 \%$ of the time. The mean of 10,000 Lindley model parameter estimate and the $95 \%$ CI obtained by computing the 2.5 and 97.5 percentiles of the distribution of the estimates are $0.10259(0.074672$, $0.14044)$ for the composition method; $0.10241(0.074687,0.13973)$ for the inverse transform method. For composition method with $n=20, \theta=9$, the center of the CI (i.e., parameter estimate) is above the true parameter value $53.16 \%$ of the time; for the inverse transform method, the parameter estimate is above the true parameter value $52.73 \%$ of the time. The mean of 10,000 Lindley model parameter estimate and the $95 \%$ CI obtained by computing the 2.5 and 97.5 percentiles of the distribution of the estimates are $9.42(6.256,14.212)$ for the composition method; $9.404(6.264,14.239)$ for the inverse transform method. Figures 3 and 4 present the distribution of the parameter estimates for the 10,000 replicates each with sample size of 20 from the two methods. The distribution of the estimates are similar.

Table 5 presents the estimated size of tests (false positive rates or type I error rates) for comparing the two EDFs from simulated data using the composition and the inverse transform methods. In all combinations of parameter values and sample sizes studied, no evidence exist to suggest a difference in the empirical distribution functions based on the two methods of generating the data that follow the Lindley distribution. That is, all $\hat{\alpha}$ are $\leq 0.05$, where $\hat{\alpha}$ represents the proportion (in percent) of times the null hypothesis is rejected out of $m$ replicates. The false positive rate from the K-S appears to be conservative (i.e., less than 0.05). This might be because if parameters are estimated from the sample to provide a control distribution against which the sample is to be tested, the actual significance level of rejection is smaller that the selected significance level (Crutcher, 1975).

Generating random variables using these two methods is not restricted to the Lindley distribution; they may be applied to other distributions. As noted in the introduction, the choice of a method is contextual. The composition method may be utilized for settings when distributions are not supported by software, and it is more efficient or convenient to expressed a PDF as a mixture of component densities. If the PDF is supported by existing software, there may be no gain in looking for other 


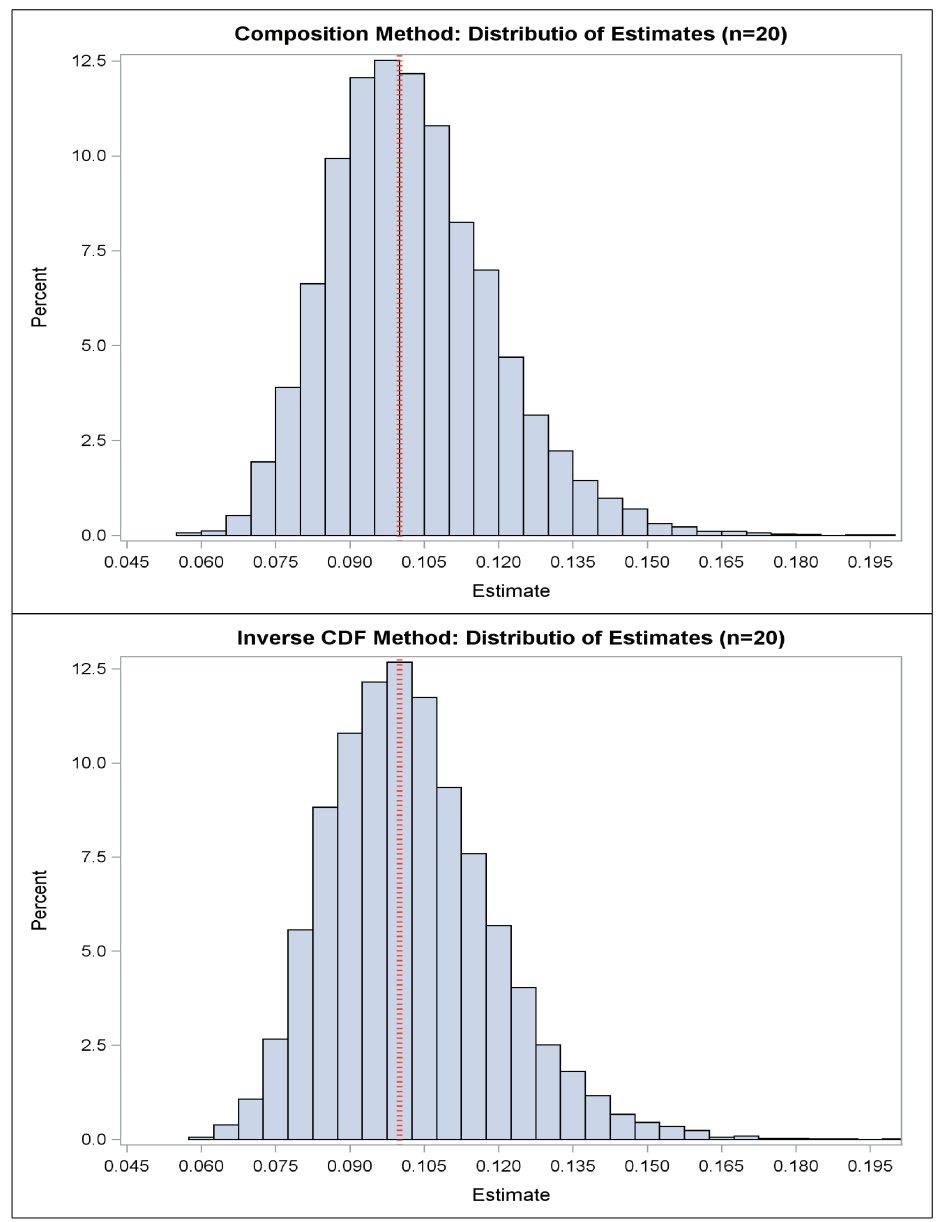

Figure 3: Distribution of 10,000 Lindley model parameter estimates $(n=20 ; \theta=0.1)$.

approaches to generate the distribution. Although non-existence in closed form of inverse transformation of a distribution function does not preclude generation of a random variates using the inversion approach, other factors such as computation costs and convenience should be considered. If a computationally more efficient approach exists, such an approach should be used.

For $n=10,000 ; \theta=0.1$, using composition method, our implementation took 6 seconds to run on a personal computer with a $2.50 \mathrm{GHz}$ processor and 4.0 GB RAM; for the inverse transform method using Newton's numerical technique, it took 11 seconds.

\section{Conclusions}

The results from this study do not support the assertions that generating data from the Lindley model using inversion method fails because $F(t)=U$ cannot be explicitly expressed. Essentially the same results are obtained on statistical properties of the MLE when data that follow the Lindley distribution are generated using the composition and the inverse transform methods. Having no closed form for 


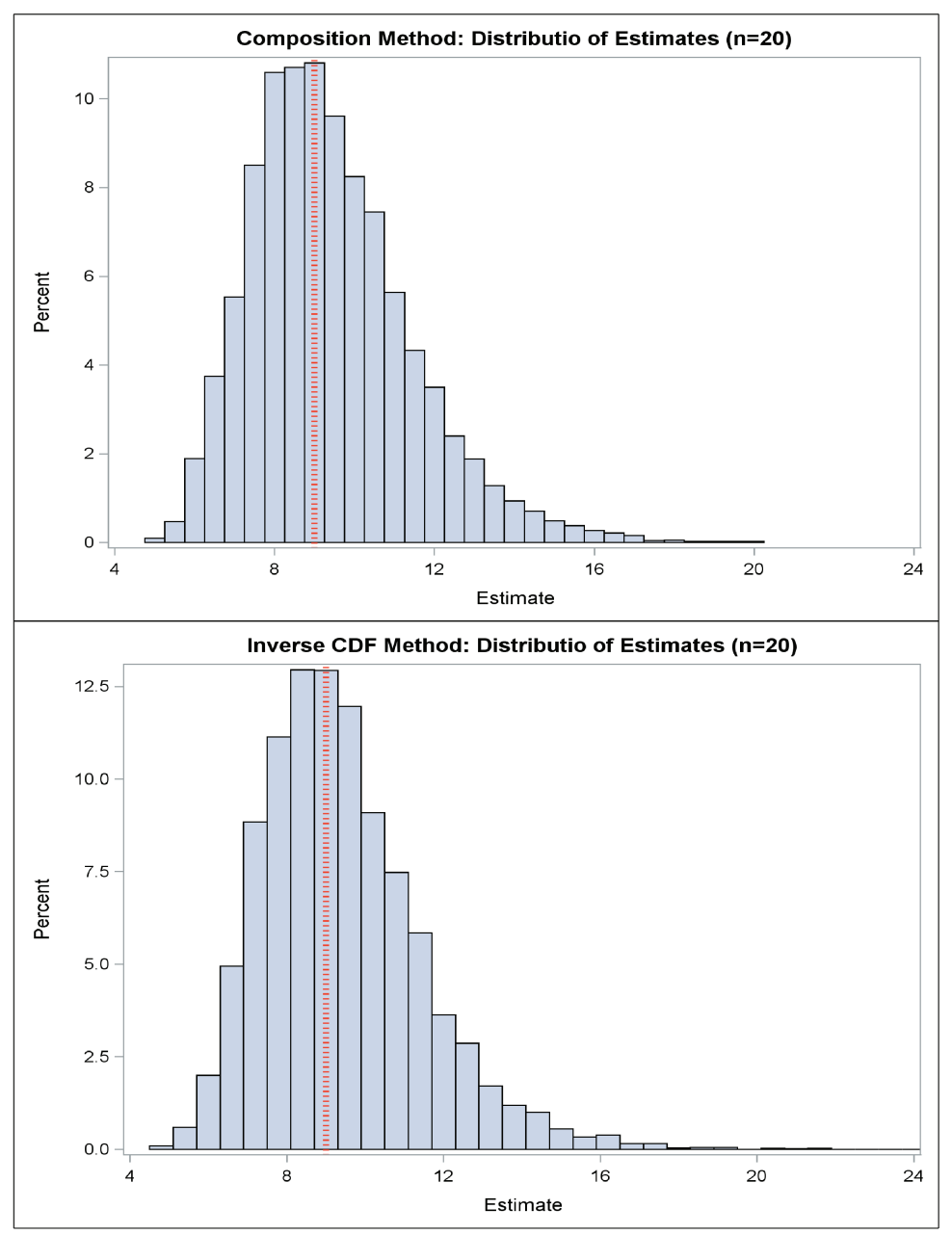

Figure 4: Distribution of 10,000 Lindley model parameter estimates $(n=20 ; \theta=9)$.

Table 5: Size of test for comparing empirical distribution functions based on simulated data using composition and inverse transform methods

\begin{tabular}{ccccccc}
\hline \hline \multicolumn{7}{c}{$H_{0}: F_{1}=F_{2}$} \\
\hline \multirow{2}{*}{$\theta$} & Par & \multicolumn{5}{c}{ Sample size $(n)$} \\
\cline { 2 - 7 } & $\hat{\alpha}$ & 3.22 & 40 & 80 & 100 & 200 \\
\hline \multirow{2}{*}{0.1} & $\mathrm{CI}$ & $2.88,3.58$ & $2.81,3.50$ & $3.37,4.12$ & $3.40,4.15$ & $3.52,4.29$ \\
\hline \multirow{2}{*}{1.0} & $\hat{\alpha}$ & 3.26 & 3.27 & 3.61 & 3.70 & 3.00 \\
& $\mathrm{CI}$ & $2.92,3.63$ & $2.93,3.64$ & $3.25,3.99$ & $3.34,4.09$ & $3.62,4.40$ \\
\hline \multirow{2}{*}{9.0} & $\hat{\alpha}$ & 3.32 & 3.31 & 3.38 & 3.83 & 4.16 \\
& $\mathrm{CI}$ & $2.98,3.69$ & $2.97,3.68$ & $3.03,3.75$ & $3.46,4.22$ & $3.78,4.57$ \\
\hline \hline
\end{tabular}

Values are $\hat{\alpha} \times 100$, where $\hat{\alpha}$ represents the proportion of times the null hypothesis is rejected out of $m$ replicates.

The null hypothesis is that the EDF of data simulated using the composition $\left(F_{1}\right)$ and inverse transform $\left(F_{2}\right)$ methods are equal. $\mathrm{CI}=$ Monte Carlo exact $95 \%$ confidence interval. 


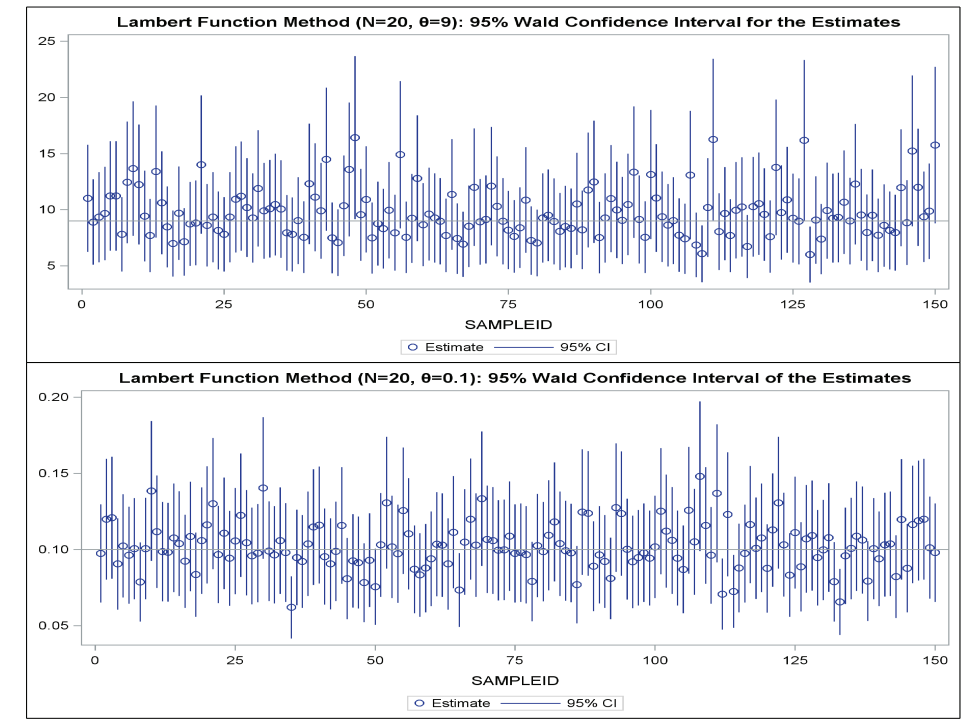

Figure A.1: Wald-based 95\% confidence intervals $(n=20 ; \theta=9$ and $\theta=0.1)$.

$F(t)=U$ does not preclude generation of data from the Lindley distribution using inverse transform method-the same is true for any distribution.

\section{Appendix A: Generating data from Lindley distribution using Lambert function}

The Lambert function $W(z)$ is an inverse function (Chapeau-Blondeau and Monir, 2002). Jodrá (2010) expressed the quantile function of the Lindley random variable as Lambert function. Briefly, set $Z(p)=-1-\theta-\theta Q(p)$; then

$$
\begin{aligned}
\frac{\exp (1+\theta)}{1+\theta}(-Z(p) \exp (Z(p))) & =1-p, \\
Z(p) \exp (Z(p)) & =\frac{-(1+\theta)(1-p)}{\exp (1+\theta)}, \\
Z(p) & =W_{-1}\left(\frac{-(1+\theta)(1-p)}{\exp (1+\theta)}\right), \quad 0<p<1,
\end{aligned}
$$

where $W_{-1}$ is the negative branch of Lambert function. Hence,

$$
\begin{aligned}
-1-\theta-\theta Q(p) & =W_{-1}\left(\frac{-(1+\theta)(1-p)}{\exp (1+\theta)}\right), \\
Q(p) & =-1-\frac{1}{\theta}-\frac{1}{\theta} W_{-1}\left(\frac{-(1+\theta)(1-p)}{\exp (1+\theta)}\right) .
\end{aligned}
$$

For data generated using the Lambert function, Figure A.1 presents the Wald-based $95 \%$ confidence intervals (CIs) of the parameter estimates for the first 150 replicates for $\theta=0.1,9$; and $n=20$. The Wald-based $95 \%$ CIs of the parameter estimates from the Lindley distribution generated using the Lambert function are similar to those from the Lindley distribution generated using the composition and the inverse transform methods (Figures 1 and 2). Providing the $95 \%$ CIs for the parameter estimates for all the replicates is impractical because of space limitation. 


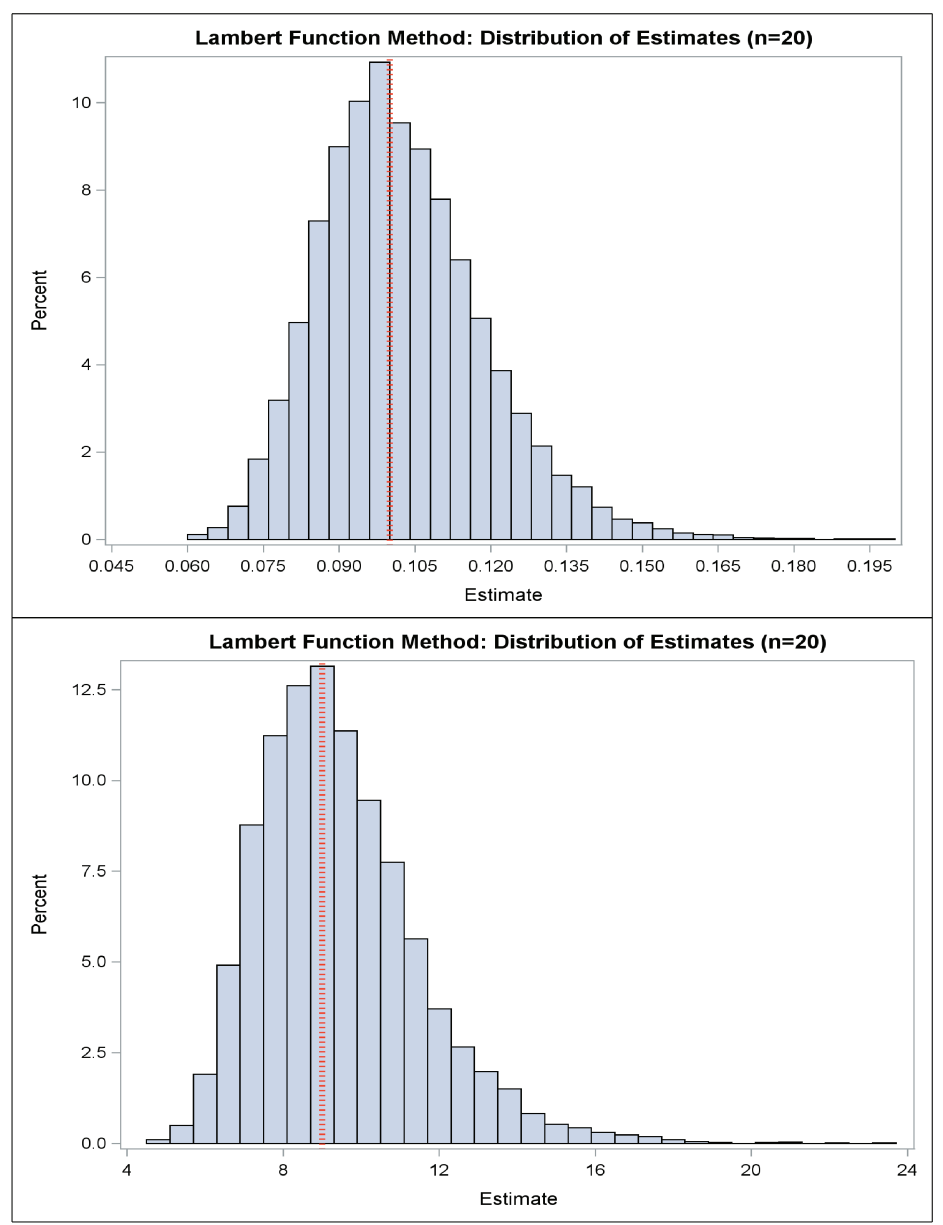

Figure A.2: Distribution of 10,000 Lindley parameter estimates $(n=20 ; \theta=9$ and $\theta=0.1)$.

For Lambert function method with $n=20, \theta=9$, the center of the $95 \%$ CI (i.e., parameter estimate) is above the true parameter value $53.16 \%$ of the time; for $\theta=0.10$, the parameter estimate is above the true parameter value $51.62 \%$ of the time. The mean of 10,000 Lindley model parameter estimate and the $95 \%$ CI obtained by computing the 2.5 and 97.5 percentiles of the distribution of the estimates are $9.4366(6.300,14.318)$ for $\theta=9 ; 0.1025(0.0750,0.1391)$ for $\theta=0.1$.

Figure A.2 displays the distribution of 10,000 parameter estimates of the Lindley distribution.

\section{References}

Agresti A, Wackerly D, and Boyett JM (1979). Exact conditional tests for cross-classifications: approximation of attained significance levels, Psychometrika, 44, 75-83.

Banks J (1998). Handbook of Simulation: Principles, Methodology, Advances, Applications, and Practice, John Wiley \& Sons, New York. 
Bender R, Augustin M, and Blettner M (2005). Generating survival times to simulate Cox's proportional Hazards models, Statistics in Medicine, 24, 1713-1723.

Chapeau-Blondeau F and Monir A (2002). Numerical evaluation of the Lambert W function and application to generation of the generalized Gaussian noise with exponent 1/2, IEEE Transactions on Signal Processing, 50, 2160-2165.

Cox DR (1972). Regression models and life-tables, Journal of the Royal Statistical Society Series B (Methodological), 34, 187-220.

Crutcher HL (1975). A note on possible miseuse of the Kolmogorov-Smirnov test, Journal of Applied Meteorology, 14, 1600-1603.

Ghitany ME, Atieh B, and Nadarajah S (2008). Lindley distribution and its application, Mathematics and Computers in Simulation, 78, 493-506.

Jodrá P (2010). Computer generation of random variables with Lindley or Poisson-Lindley distribution via the Lambert W function, Mathematics and Computers in Simulation, 81, 851-859.

Lindley DV (1958). Fiducial distributions and Baye's theorem, Journal of the Royal Statistical Society Series B (Methodological), 20, 102-107.

Okwuokenye M. (2012). Size and power of tests of hypotheses on parameters when modelling timeto-event data with the Lindley distribution (Doctoral dissertation), Jiann-Ping Hsu College of Public Health, Georgia Southern University, Statesboro, GA.

Okwuokenye M and Peace KE (2015a). A comparison of size and power of tests of hypotheses on parameters based on two generalized Lindley distributions, Communications for Statistical Applications and Methods, 22, 233-239.

Okwuokenye M and Peace KE (2015b). Size and power of tests of hypotheses on survival parameters from the Lindley distribution with covariates, Austin Biometrics and Biostatistics, 2, 1-6.

Peace KE and Flora RE (1978). Size and power assessment of tests of hypotheses on survival parameters, Journal of the American Statistical Association, 73, 129-132.

Qian J, Li B, and Chen PY (2010). Generating survival data in the simulation studies of Cox's model. In Proceedings of 3rd International Conference on Information and Computing, Wuxi, China, 93-96.

Wicklin R (2013). Simulating Data with SAS, SAS Institute Inc, Cary, NC. 